\title{
Oil-mediated high-throughput generation and sorting of water-in-water droplets
}

\author{
Lang Nan', Yang Cao', Shuai Yuan² and Ho Cheung Shum ${ }^{1}$
}

\begin{abstract}
Aqueous two-phase system (ATPS) droplets have demonstrated superior compatibility over conventional water-in-oil droplets for various biological assays. However, the ultralow interfacial tension hampers efficient and stable droplet generation, limiting further development and more extensive use of such approaches. Here, we present a simple strategy to employ oil as a transient medium for ATPS droplet generation. Two methods based on passive flow focusing and active pico-injection are demonstrated to generate water-water-oil double emulsions, achieving a high generation frequency of $\sim 2.4 \mathrm{kHz}$. Through evaporation of the oil to break the double emulsions, the aqueous core can be released to form uniform-sized water-in-water droplets. Moreover, this technique can be used to fabricate aqueous microgels, and the introduction of the oil medium enables integration of droplet sorting to produce singlecell-laden hydrogels with a harvest rate of over $90 \%$. We believe that the demonstrated high-throughput generation and sorting of ATPS droplets represent an important tool to advance droplet-based tissue engineering and single-cell analyses.
\end{abstract}

\section{Introduction}

Droplet microfluidics has demonstrated vast promise in processing and analyzing biological samples due to its capacity to confine individual targets in microscale volumes and perform high-throughput manipulations ${ }^{1-4}$. However, the conventional use of water-oil droplet systems is constrained by the toxic nature of organic reagents, which reduces their biocompatibility and prevents access to droplet contents ${ }^{5}$. With an all-aqueous environment, water-in-water droplets produced by introducing aqueous two-phase systems (ATPSs) have attracted growing interest and shown great potential in fabricating biomimetic architectures ${ }^{6,7}$.

ATPS is typically an aqueous solution of two incompatible polymers or one polymer and one salt, which phase-separates to form two immiscible phases when the

Correspondence: Ho Cheung Shum (ashum@hku.hk)

'Department of Mechanical Engineering, The University of Hong Kong, Pokfulam Road, Hong Kong, China

${ }^{2}$ Department of Electrical and Electronic Engineering, The University of Hong Kong, Pokfulam Road, Hong Kong, China

These authors contributed equally: Lang Nan, Yang Cao concentrations exceed their corresponding critical values $^{8}$. The potential biocompatibility has promoted ATPSs as a promising candidate for various biological assays, including cell culture ${ }^{9}$, protein delivery ${ }^{10}$, and DNA partitioning ${ }^{11}$, but a strategy to generate water-inwater droplets is needed. To form droplets with uniform shapes and sizes, the two phase-separated immiscible phases are conventionally introduced into microfluidic channels for droplet formation ${ }^{12}$. However, interfaces of two aqueous phases can possess ultralow interfacial tension (typically in the range of $10^{-4} \sim 10^{-1} \mathrm{mN} / \mathrm{m}$ ), challenging the efficient and stable generation of droplets in microfluidic systems: The droplets can be formed only under ultralow flow rates (below $3 \mu \mathrm{L} / \mathrm{h}$ ) at frequencies below $10 \mathrm{~Hz}$, and the droplet size distribution is also undesirably large ${ }^{13}$. Moreover, when polyelectrolytes or nanoparticles are added to stabilize the droplets, their fast assembly at the interface hampers pinch-off of the interface, further lowering the generation efficiency ${ }^{14,15}$.

To improve the droplet generation efficiency, active actuation strategies have been employed; by providing additional energy, pinch-off of the interfaces can be 
triggered. For instance, with the assistance of acoustic vibration and oil chopping to destabilize the jet, the generation frequency can be increased two-fold in comparison to passive generation in glass-capillary devices $^{16,17}$. Polydimethylsiloxane (PDMS) devices with typically two-dimensional planar geometries are often used, where more sophisticated droplet manipulation is enabled, for instance, droplet sorting ${ }^{18}$ and picoinjection $^{19}$. However, the shear force in these geometries dampens the active actuation, which becomes less effective in generating droplets with a high throughput ${ }^{20}$. As a result, even with mechanical actuation and eight parallel channels, the highest production rate is only approximately $100 \mathrm{~Hz}$, which is still inefficient compared with water-in-oil droplet generation, typically with rates on the order of $\mathrm{kHz}^{21}$. Moreover, due to the similar electrical properties of the two aqueous phases, electrical manipulations of the generated droplets, such as dielectrophoretic (DEP)-based droplet sorting ${ }^{22}$, fail to work and preclude certain sample processing and analysis steps.

To address these problems, we propose an oil-mediated strategy to generate ATPS droplets, where water-water-oil double emulsions are first generated, with the inner aqueous cores subsequently released to form water-inwater droplets. To demonstrate the efficiency of this approach, double emulsions are passively generated in a flow-focusing channel at high throughput and with a uniform size distribution. The use of a transient oil medium also enables electric actuation to further increase the droplet generation rate and degree of control of the generated droplets. To extract the inner aqueous droplets, the transient oil layer is evaporated in a gentle and efficient manner. Through further incorporation of sodium alginate and integration of a droplet sorting system, single-cell-laden hydrogels can be produced, with a high harvest rate.

\section{Results}

\section{Passive double-emulsion generation}

As the first step in ATPS droplet formation, the generation of water-water-oil double emulsions determines the final droplet size and morphology. To generate monodisperse double-emulsion drops, we passively break up the aqueous flow into droplets by oil pinching, which can be achieved in a typical multi-inlet flow-focusing channel $^{23}$. Different from the water-oil-water (W-O-W) double-emulsion drops that are formed by two steps of emulsification $^{24}$, water-water-oil (W-W-O) doubleemulsion drops are formed in a single step at the second cross junction: The two aqueous phases flow laminarly side-by-side after meeting in the first junction, followed by pinch-off of the water-water compound jet by the oil flow to form W-W-O double-emulsion drops (Fig. 1a and Movie S1). To demonstrate the efficiency of this method, the two most commonly used ATPS polymers, polyethylene glycol (PEG) and dextran (DEX) that are both biocompatible, are introduced. To prevent coalescence and destabilization of the droplets during subsequent droplet extraction, two oppositely charged polyelectrolytes, poly(allylamine hydrochloride) (PAH) and poly(sodium 4-styrenesulfonate) (PSS), are dissolved into the innermost (DEX-rich) and intermediate (PEGrich) solutions to stabilize the water-water interface through interfacial complexation. However, due to the fast reaction, premature precipitation prior to droplet breakup tends to gradually block the channel and cause a transition from the original dripping regime to an unstable jetting regime within $3 \mathrm{~min}$. Thus, a spacing stream of pure PEG-rich solution is introduced at a flow rate of $100 \mu \mathrm{L} / \mathrm{h}$ to separate the two reagent streams, thereby enabling stable droplet generation for over $1 \mathrm{~h}$ and forming monodisperse double emulsions (Fig. 1b, c).

To optimize the microfluidic operations for controllable double-emulsion generation, the droplet size and generation frequency at different flow conditions are systematically studied. In this flow regime, the two streams of PEG-rich phases with and without PSS converge into one stream before droplet generation, and varying the flow rate ratio between the two PEG-rich phases does not lead to any observable effect on the resultant droplet size (Fig. S1). Thus, in the following analysis, we use the combined flow rate to characterize the flow behaviors. First, by regulating the flow rates of the aqueous phases, the formed core-shell structure can be controlled. We change the flow rate ratio of the DEX-rich phase and PEG-rich phase from 1:7 to 7:1 while keeping the oil flow rate constant. As a result, the inner droplet diameter increases while the outer droplet diameter remains unchanged, with the diameter ratio ranging from 50 to $96 \%$ and the resultant shell thickness varying from $26 \mu \mathrm{m}$ to $\sim 2 \mu \mathrm{m}$ (Fig. 1d). The flow rate of oil is a key parameter that affects both the double-emulsion drop size and generation frequency. To quantify the effect of flow rate on the double-emulsion formation, we set the flow rates of DEXrich and PEG-rich phases constant at $200 \mu \mathrm{L} / \mathrm{h}$ and $600 \mu \mathrm{L} / \mathrm{h}$, respectively, while tuning the flow rate of oil from 2000 to $10,000 \mu \mathrm{L} / \mathrm{h}$. For oil flow rates below $2000 \mu \mathrm{L} / \mathrm{h}$, the aqueous compound jet cannot be pinched off. Experimentally, the core diameter gradually drops from $73 \mu \mathrm{m}$ to $\sim 43 \mu \mathrm{m}$ as the oil flow rate increases, and the generation frequency increases from $270 \mathrm{~Hz}$ to $\sim 1200 \mathrm{~Hz}$ (Fig. 1e). Since the two aqueous phases are formed by phase separation of PEG and DEX solutions, the adopted polymer concentrations determine the interfacial tension and viscosity of the two aqueous phases, thus affecting the interface pinch-off and droplet breakup. To understand the effect of polymer concentrations and test the feasibility of this approach in 

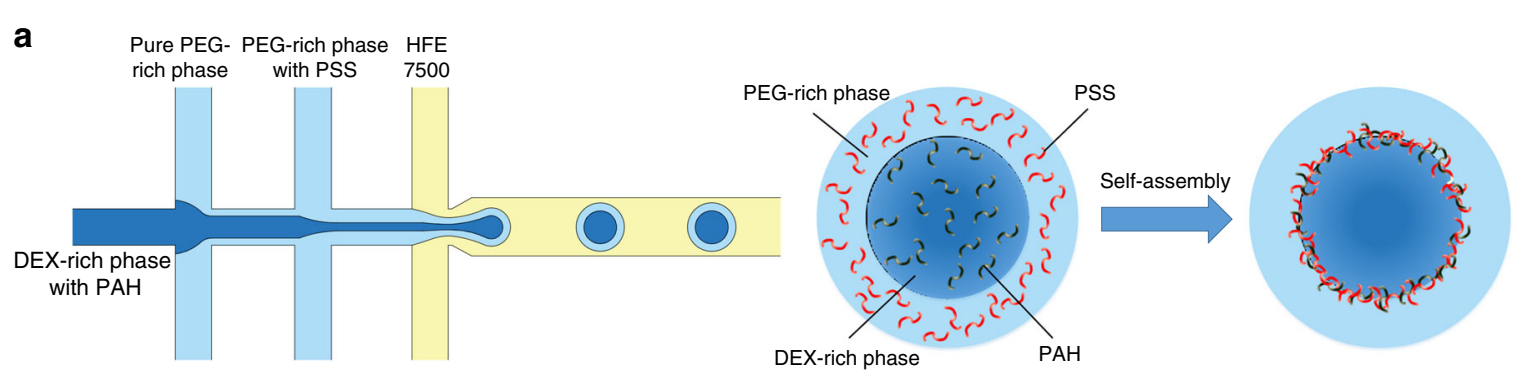

b

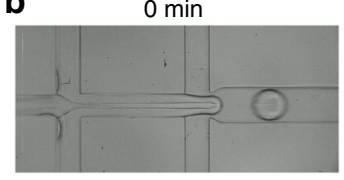

$0 \min$

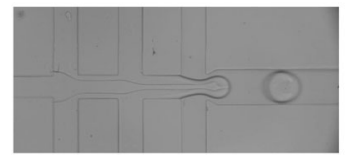

d

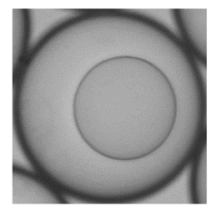

Flow-rate ratio of 1:7

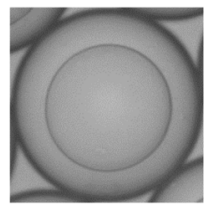

$1: 3$

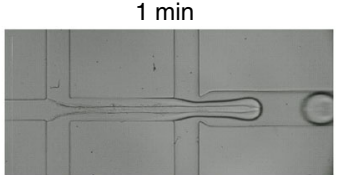

$30 \mathrm{~min}$
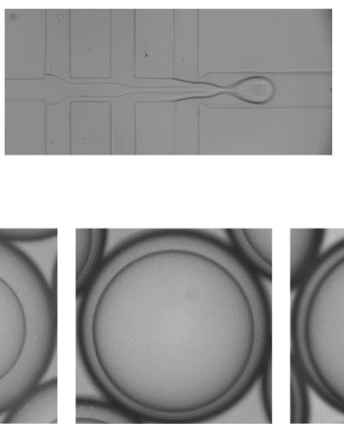

$1: 1$

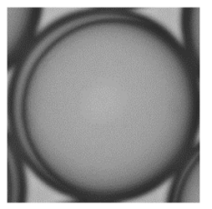

$3: 1$
$3 \min$

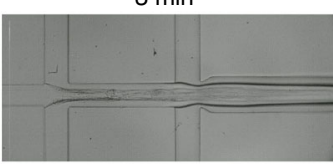

$60 \mathrm{~min}$
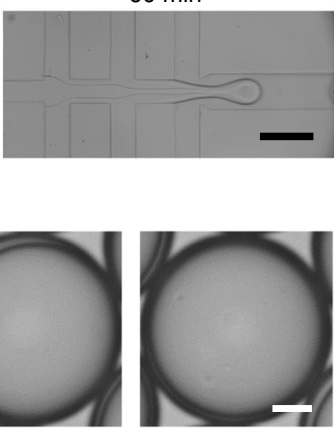

$7: 1$ c

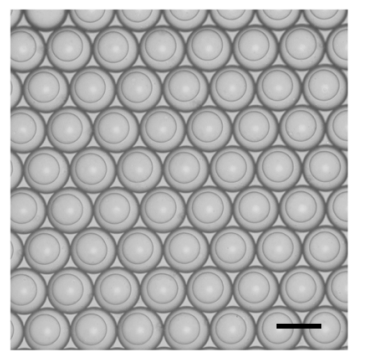

$\mathbf{e}_{75}$

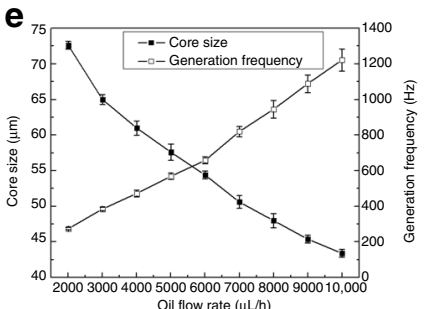

Fig. 1 Passive generation of water-water-oil double emulsions. a Schematic of the passive method to generate water-water-oil double emulsions in a multi-inlet flow-focusing channel. b Comparison of the double-emulsion generation without and with a spacing stream. Scale bar: $200 \mu \mathrm{m}$. c Image of the double-emulsion drops generated at flow rates of $200 \mu \mathrm{L} / \mathrm{h}$ (DEX-rich), $600 \mu \mathrm{L} / \mathrm{h}$ (PEG-rich) and $3000 \mu \mathrm{L} / \mathrm{h}$ (oil). Scale bar: $100 \mu \mathrm{m}$. d The morphology of double-emulsion drops formed at different core-shell flow-rate ratios. The flow rate of oil is set constant at $3000 \mu \mathrm{L} / \mathrm{h}$, while the flow rates of DEX-rich:PEG-rich phases are 100:700, 200:600, 400:400, 600:200 and 700:100 $\mu \mathrm{L} / \mathrm{h}$, respectively. Scale bar: $20 \mu \mathrm{m}$. e A plot of the core droplet diameter and generation frequency as the oil flow rate increases from 2000 to 10,000 $\mu \mathrm{L} / \mathrm{h}$

more viscous flows, we increase the PEG and DEX concentrations from 10 to $20 \%$ and measure the resultant core size and generation frequency at constant flow conditions $(200 \mu \mathrm{L} / \mathrm{h}$ for the DEX-rich phase, $600 \mu \mathrm{L} / \mathrm{h}$ for the PEG-rich phase and $3000 \mu \mathrm{L} / \mathrm{h}$ for the oil phase). The results indicate that as the polymer concentration increases, the size of the core droplets increases from $65 \mu \mathrm{m}$ to $77 \mu \mathrm{m}$, and the frequency decreases from 385 to $240 \mathrm{~Hz}$ (Tables 1 and 2). In summary, this method enables high-throughput generation of W-W-O double emulsions with controlled structures.

\section{Active double-emulsion generation}

In addition to the demonstrated passive generation, double-emulsion drops can be actively generated by injecting the core droplet into the shell droplet one-byone by pico-injection. Here, a typical pico-injection channel is employed for the two-step generation ${ }^{25}$. In the flow-focusing junction, the PEG-rich solution is broken up into droplets under the shear effect of the oil stream. Then, when the droplets flow by the pico-injector, a high voltage is applied to the electrodes to destabilize the water-oil interface, thus introducing the DEX-rich solution into the shell drop to form a double emulsion (Fig. 2a). This method relies on accurate control over the applied electric field, where an optimal pulse with a frequency of $10 \mathrm{kHz}$ and peak-to-peak voltage of $500 \mathrm{~V}_{\mathrm{p}-\mathrm{p}}$ is applied to achieve a stable injection with identical volumes injected into each droplet ${ }^{25}$.

In comparison with the passive generation that simultaneously pinches off two aqueous streams, only one stream of the PEG-rich phase is segmented in the active operations, thus allowing more frequent double-emulsion generation. To demonstrate the higher efficiency, a similar test is performed to explore the effect of the oil flow rate on the double-emulsion generation. We change the flow rate of the oil phase from 500 to $10000 \mu \mathrm{L} / \mathrm{h}$, while the flow rates of the droplet phase (PEG-rich) and 
injection phase (DEX-rich) are maintained at 600 and $200 \mu \mathrm{L} / \mathrm{h}$, respectively. The results indicate that under the same flow conditions, smaller droplets can be generated at a higher frequency in the active mode. For instance, at the same oil flow rate of $3000 \mu \mathrm{L} / \mathrm{h}$, the frequency of active generation $(750 \mathrm{~Hz})$ is approximately two times that of passive generation $(380 \mathrm{~Hz})$ (Fig. 2b). Moreover, doubleemulsion formation at different polymer concentrations is studied. We set the flow rates of DEX-rich, PEG-rich and oil phases at 200, 600 and $3000 \mu \mathrm{L} / \mathrm{h}$, respectively.

Table 1 The density, viscosity and interfacial tension of different concentrations of polymer solutions

\begin{tabular}{llll}
\hline $\begin{array}{l}\text { Polymer } \\
\text { solutions }\end{array}$ & $\begin{array}{l}\text { Density (g/ } \\
\left.\mathbf{c m}^{3}\right)\end{array}$ & $\begin{array}{l}\text { Viscosity } \\
\mathbf{( P a} \cdot \mathbf{s})\end{array}$ & $\begin{array}{l}\text { Interfacial } \\
\text { tension }(\mathbf{m N} / \mathbf{m})\end{array}$ \\
\hline PEG-rich 10\% & 1.038 & 0.011 & 0.116 \\
DEX-rich 10\% & 1.100 & 0.018 & \\
PEG-rich 10\% & 1.036 & 0.014 & 0.157 \\
DEX-rich 15\% & 1.130 & 0.030 & \\
PEG-rich 10\% & 1.044 & 0.020 & 0.186 \\
DEX-rich 20\% & 1.135 & 0.032 & \\
PEG-rich 15\% & 1.039 & 0.018 & 0.173 \\
DEX-rich 10\% & 1.127 & 0.033 & \\
PEG-rich 15\% & 1.044 & 0.027 & 0.249 \\
DEX-rich 15\% & 1.151 & 0.052 & \\
PEG-rich 15\% & 1.048 & 0.034 & 0.394 \\
DEX-rich 20\% & 1.165 & 0.072 & \\
PEG-rich 20\% & 1.046 & 0.035 & 0.368 \\
DEX-rich 10\% & 1.166 & 0.083 & \\
PEG-rich 20\% & 1.050 & 0.044 & 0.478 \\
DEX-rich 15\% & 1.179 & 0.123 & \\
PEG-rich 20\% & 1.054 & 0.060 & 0.521 \\
DEX-rich 20\% & 1.195 & 0.154 & \\
\hline
\end{tabular}

Experimentally, when the concentrations of the two polymers increase from 10 to $20 \%$, the resultant core size increases from 52 to $61 \mu \mathrm{m}$, and the generation frequency decreases from 750 to $480 \mathrm{~Hz}$ (Table 3).

In addition to the higher efficiency, the active generation method allows injection of different ingredients into the core droplets, thereby enabling time-controlled reactions within the droplets. We achieve this aim in a dualinjector device, where the two injectors are closely located at a spacing of $500 \mu \mathrm{m}$ to efficiently merge the injected cores before the assembly of polyelectrolytes to form a membrane at the interface. To demonstrate the efficiency of this approach, a fluorescently labeled DEX-rich solution is introduced from the first injector, while a conventional solution without fluorescent dye is introduced from the second injector (Fig. 2c). As the flow rate ratio of the two injection flows is tuned from 1:7 to 7:1, double emulsions with different fluorescence intensities can be formed, achieving good mixing to allow accurate control of the fluorescent polyelectrolyte concentration (Fig. 2d).

\section{Core-droplet extraction}

To extract the encapsulated core droplets for ATPS droplet formation, the carrier oil must be removed to break the double-emulsion drops. Typically, the droplets are broken by first adding the demulsifier to destabilize the surfactant layer and then centrifuging the samples to separate the water and oil phases based on their density difference ${ }^{26}$. However, this method involves severe flow disturbance, which tends to disrupt both the water-oil interface and water-water interface, thus inducing undesirable coalescence of the ATPS droplets. Therefore, gentler extraction should be employed to break the outer droplet interface while keeping the inner droplets intact. Here, we introduce a noninvasive method that hinges on evaporation of the oil phase to break the double emulsions. This method is performed by transferring the collected emulsions into a Petri dish filled with the PEG-rich solution. When the emulsions fall onto the solution, the interfacial tension keeps the oil phase on the top, with the

Table 2 The core-droplet diameter and generation frequency at different polymer concentrations in the passive generation mode

\begin{tabular}{|c|c|c|c|c|c|c|c|}
\hline \multirow[t]{3}{*}{ DEX-rich } & & \multicolumn{6}{|c|}{$M_{w} 8000$ (PEG-rich) } \\
\hline & & \multicolumn{2}{|l|}{$10 \%$} & \multicolumn{2}{|l|}{$15 \%$} & \multicolumn{2}{|l|}{$20 \%$} \\
\hline & & Core size $(\mu \mathrm{m})$ & Frequency $(\mathrm{Hz})$ & Core size $(\mu \mathrm{m})$ & Frequency $(\mathrm{Hz})$ & Core size $(\mu \mathrm{m})$ & Frequency $(\mathrm{Hz})$ \\
\hline \multirow[t]{3}{*}{$M_{w} \quad 10,000$} & $10 \%$ & $65.0 \pm 0.7$ & $385.0 \pm 10.6$ & $66.6 \pm 0.5$ & $362.2 \pm 7.1$ & $73.4 \pm 0.5$ & $267.2 \pm 3.8$ \\
\hline & $15 \%$ & $68.2 \pm 0.4$ & $331.0 \pm 4.5$ & $72.2 \pm 0.4$ & $282.8 \pm 4.4$ & $74.6 \pm 0.5$ & $253.6 \pm 3.3$ \\
\hline & $20 \%$ & $70.2 \pm 0.4$ & $315.0 \pm 4.5$ & $74.4 \pm 0.5$ & $264.4 \pm 3.1$ & $76.6 \pm 0.5$ & $239.2 \pm 2.7$ \\
\hline
\end{tabular}


a

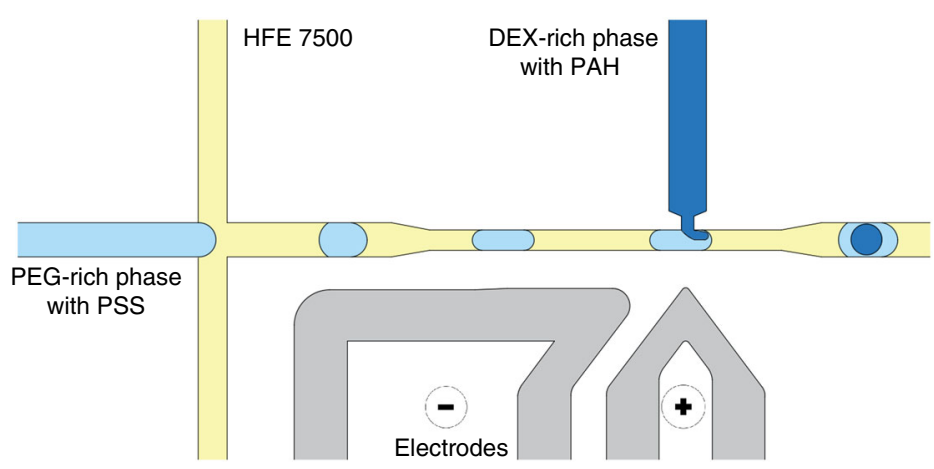

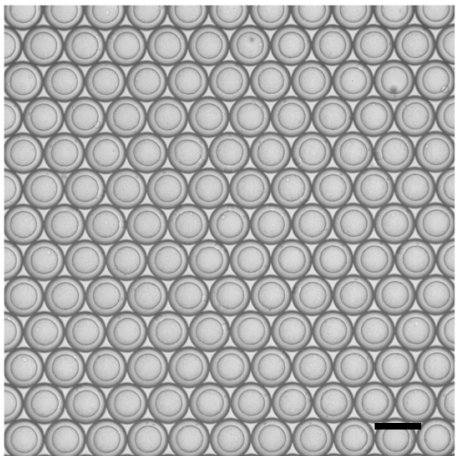

b

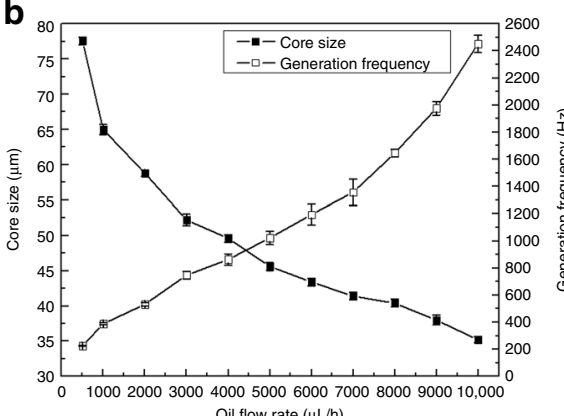

Oil flow rate $(\mu \mathrm{L} / \mathrm{h})$

d

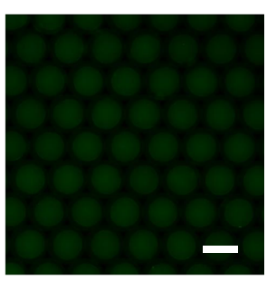

Flow-rate ratio of 1:7

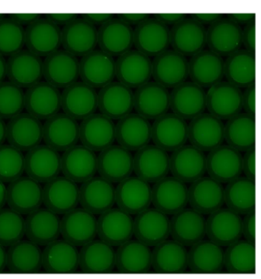

$1: 3$

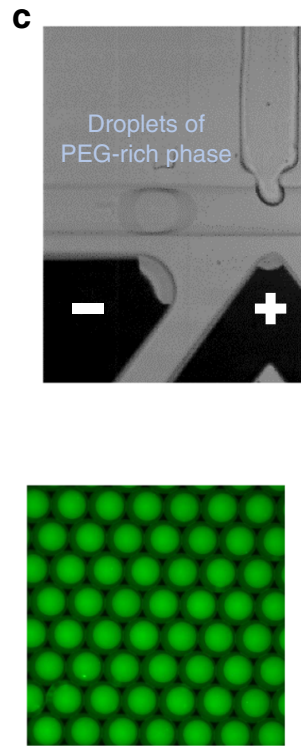

$1: 1$

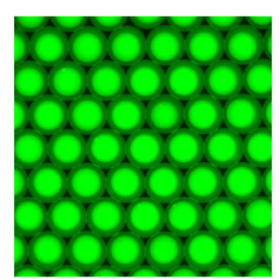

3:1

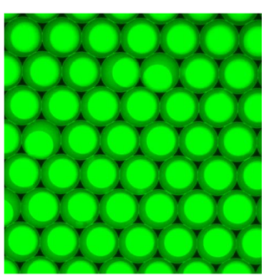

$7: 1$

Fig. 2 Active generation of water-water-oil double emulsions. a Schematic of the active method to generate water-water-oil double emulsions in a pico-injection channel and image of the double emulsions generated at flow rates of $200 \mu \mathrm{L} / \mathrm{h}$ (DEX-rich), $600 \mu \mathrm{L} / \mathrm{h}$ (PEG-rich) and $3000 \mu \mathrm{L} / \mathrm{h}$ (oil). Scale bar: $100 \mu \mathrm{m}$. b A plot of the core-droplet diameter and generation frequency as a function of the oil flow rate, which is varied from $500 \mu \mathrm{L} / \mathrm{h}$ to $10,000 \mu \mathrm{L} / \mathrm{h}$. c Image of the dual-injector device for successive injection of the DEX-rich phases with and without fluorescence. Scale bar: $100 \mu \mathrm{m}$. d Fluorescence microscopy images of the resultant double emulsions at different flow-rate ratios of the two injections. The flow rates of PEG-rich and oil phases are set constant at 600 and $3000 \mu \mathrm{L} / \mathrm{h}$, while the flow rates of the DEX-rich phases with and without fluorescence are coupled at 50:350, 100:300, 200:200, 300:100 and 350:50 $\mathrm{L} / \mathrm{h}$, respectively. Scale bar: $100 \mu \mathrm{m}$

double-emulsion drops aggregating at the center. As the surrounding oil spreads and forms a thin layer, its surface area increases. Consequently, the oil layer evaporates in $\sim 20 \mathrm{~s}$, and the shell phase merges with the PEG-rich solution underneath; hence, the cores of the DEX-rich phase are released to form monodisperse DEX-in-PEG droplets (Fig. 3a, b).

In this method, the surfactant concentration is a key factor in implementing core-droplet extraction. To elucidate the effect of the surfactant concentration on the extraction efficiency, droplets generated using different concentrations of surfactant, ranging from 0.005 to $1 \%$, are tested. The results indicate that when the surfactant concentration is below $0.02 \%$, droplet coalescence occurs due to the unstable water-oil interface; when the surfactant concentration is above $0.2 \%$, the droplets remain separated from the PEG-rich solution after complete oil evaporation (Fig. 3c, d). Thus, the surfactant concentration should be kept at appropriate ranges to stabilize the interface and release the core droplets. Using $0.05 \%$ surfactant as a demonstration, the core droplets can be efficiently extracted within $40 \mathrm{~s}$. Moreover, with this evaporation-driven extraction, the inner core droplets are free from external disturbance and can maintain their uniform shapes and sizes (coefficient of variation of approximately $2 \%)$. 
Table 3 The core-droplet diameter and generation frequency at different polymer concentrations in the active generation mode

\begin{tabular}{|c|c|c|c|c|c|c|c|}
\hline \multirow[t]{3}{*}{ DEX-rich } & & \multicolumn{6}{|c|}{$M_{w} 8000$ (PEG-rich) } \\
\hline & & \multicolumn{2}{|l|}{$10 \%$} & \multicolumn{2}{|l|}{$15 \%$} & \multicolumn{2}{|l|}{$20 \%$} \\
\hline & & Core size $(\mu \mathrm{m})$ & Frequency $(\mathrm{Hz})$ & Core size $(\mu \mathrm{m})$ & Frequency $(\mathrm{Hz})$ & Core size $(\mu \mathrm{m})$ & Frequency $(\mathrm{Hz})$ \\
\hline \multirow[t]{3}{*}{$M_{w} 10,000$} & $10 \%$ & $52.2 \pm 0.8$ & $747.0 \pm 30.1$ & $54.6 \pm 0.5$ & $641.8 \pm 23.0$ & $56.0 \pm 0.7$ & $595.4 \pm 16.5$ \\
\hline & $15 \%$ & $54.4 \pm 0.5$ & $650.2 \pm 23.0$ & $56.2 \pm 0.8$ & $595.4 \pm 16.5$ & $58.6 \pm 0.5$ & $538.0 \pm 16.4$ \\
\hline & $20 \%$ & $57.2 \pm 0.8$ & $575.2 \pm 17.5$ & $59.2 \pm 0.4$ & $520.8 \pm 11.6$ & $60.6 \pm 0.5$ & $480.8 \pm 10.7$ \\
\hline
\end{tabular}

a

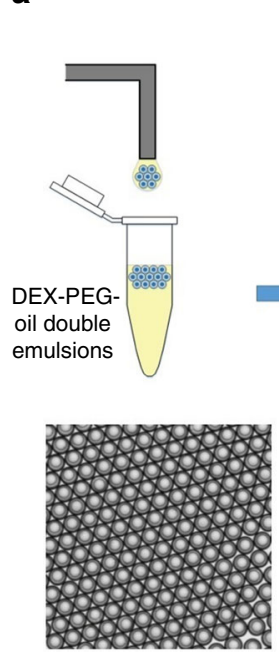

$0 \mathrm{~s}$

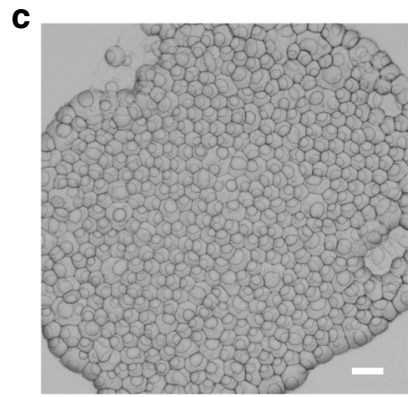

$1 \%$ surfactant

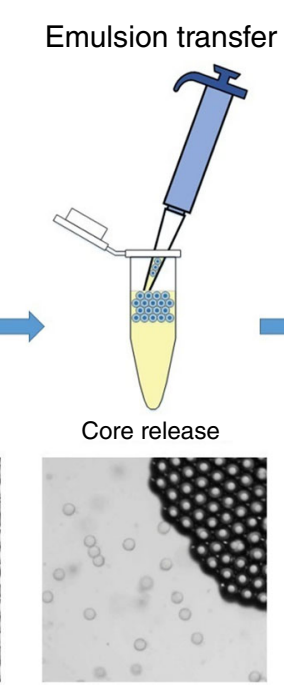

$20 \mathrm{~s}$
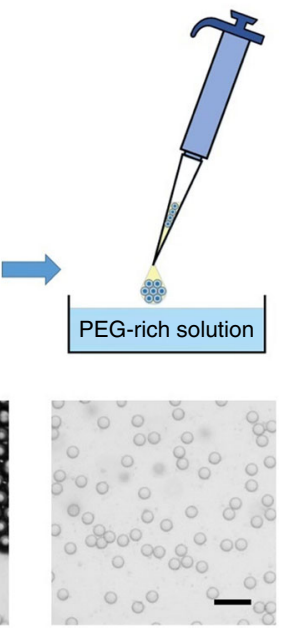

$40 \mathrm{~s}$

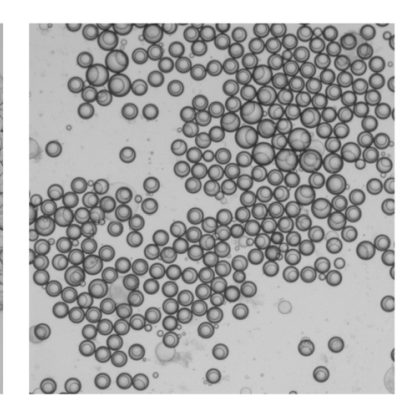

$0.01 \%$ surfactant b
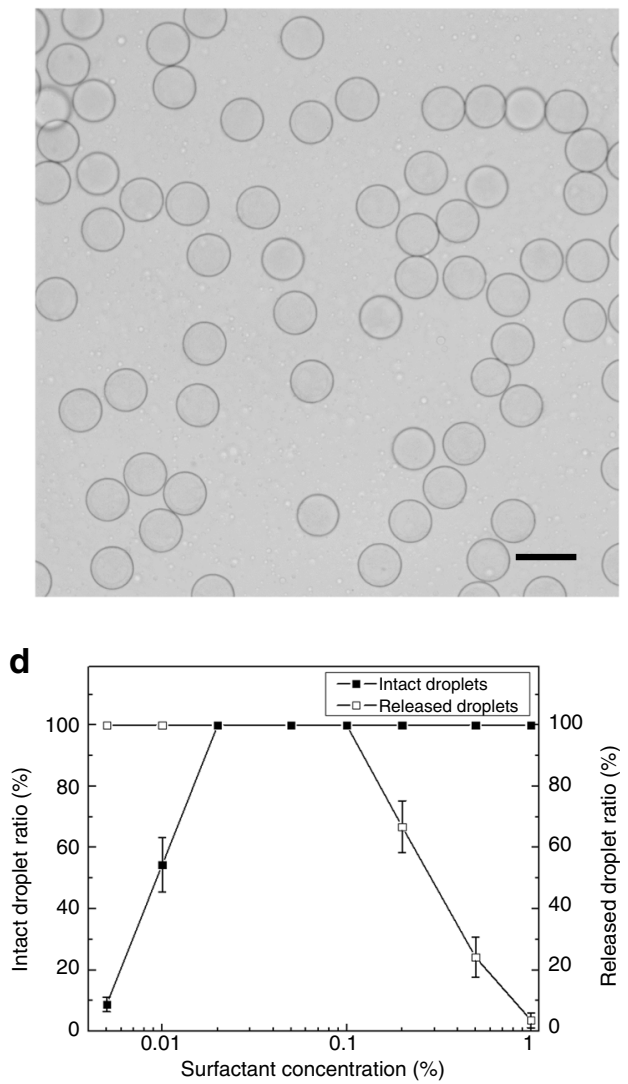

Fig. 3 Extraction of the core droplets. a Schematic showing the process of transferring the double emulsions into a PEG-rich bath and images showing the process of releasing the inner core droplets from the double emulsions. Scale bar: $200 \mu \mathrm{m}$. $\mathbf{b}$ Image of the formed monodisperse ATPS droplets using the oil-mediated method. Scale bar: $100 \mu \mathrm{m}$. c Morphology of the double emulsion during oil evaporation at surfactant concentrations of 0.01 and 1\%. Scale bar: $200 \mu \mathrm{m}$. d The chart showing the percentages of the droplets that are not coalesced and the droplets that are successfully released at different surfactant concentrations ranging from 0.005 to $1 \%$

\section{Single-cell-laden microgel sorting}

Droplet-based hydrogel microspheres, which can provide both mechanical stability and cell-binding properties, are promising scaffolds for 3D cell culture and single-cell analysis $^{27,28}$. However, the conventional fabrication method based on a water-oil system requires strong mechanical and chemical treatment to extract the formed hydrogels from organic solvents, possibly resulting in loss of cell viability ${ }^{29}$. In comparison, the demonstrated ATPS droplet facilitates biocompatible microgel fabrication in an all-aqueous 
a

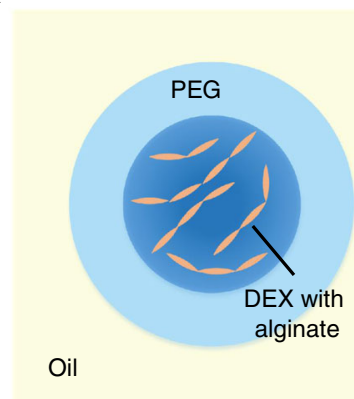

b

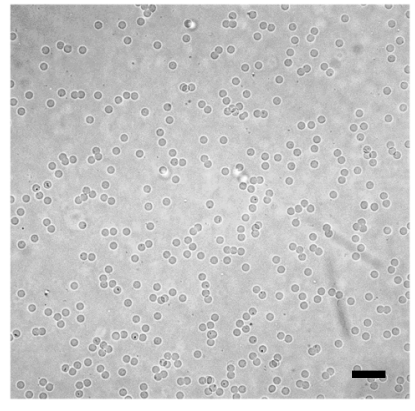

d

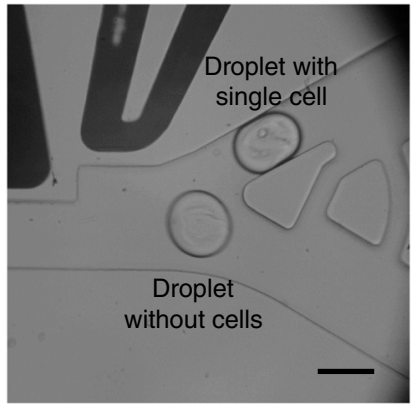

C

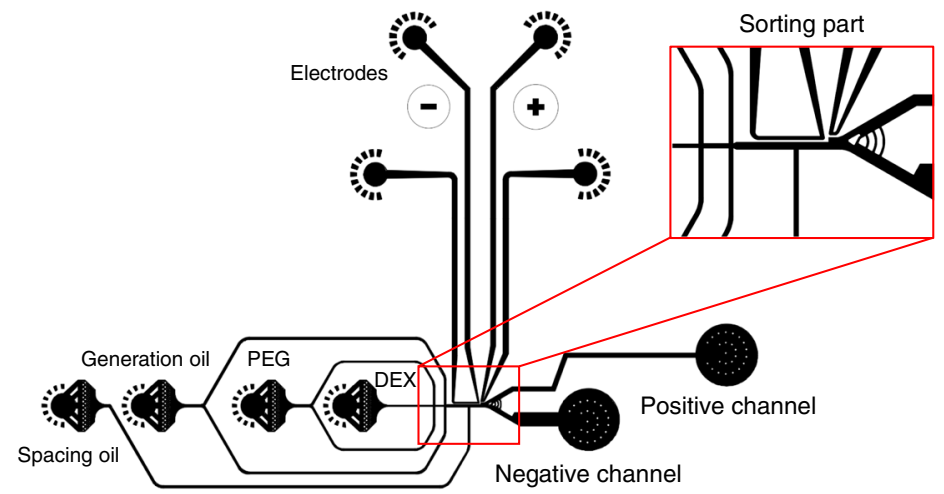

Sorting part

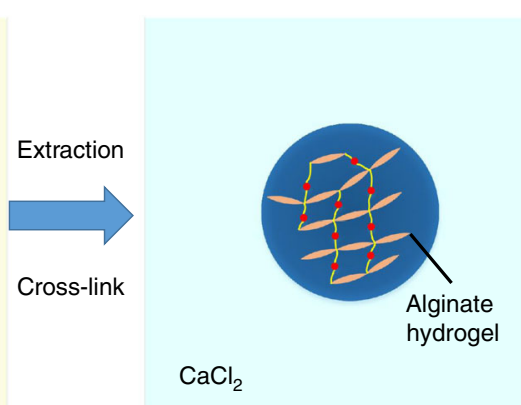

f e

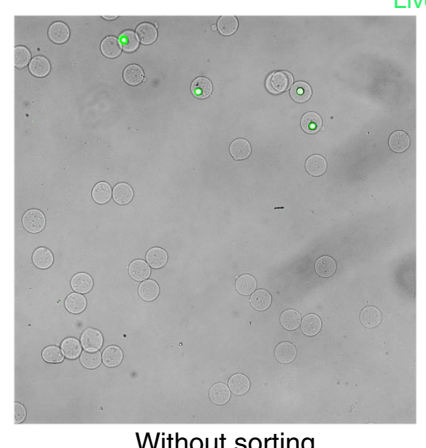

Live/Dead

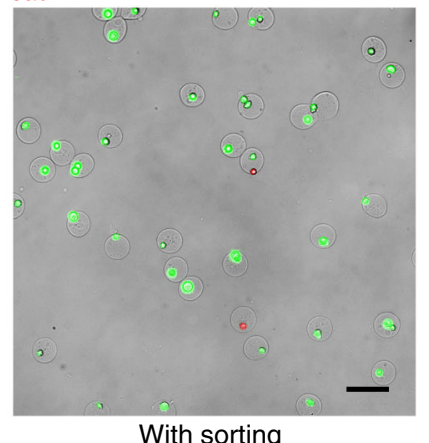

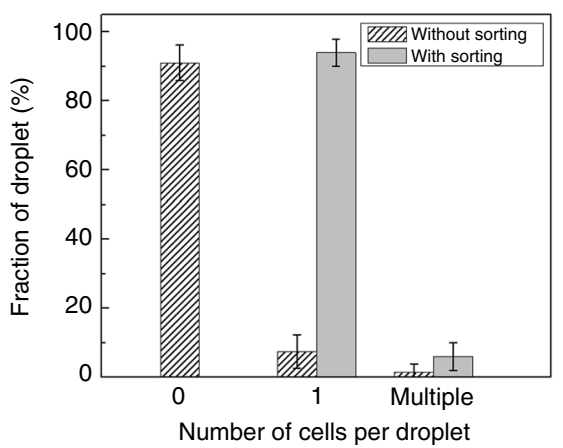

Fig. 4 Fabrication of single-cell-laden hydrogels. a Schematic showing the process of crosslinking the alginate solution by $\mathrm{Ca}^{2+}$ diffusion. $\mathbf{b}$ Image of the formed monodisperse alginate microgels. Scale bar: $200 \mu \mathrm{m}$. c Design of the integrated channel to perform continuous droplet generation and sorting. $\mathbf{d}$ Image illustrating the sorting process: The droplets with single cells are driven to the positive channel, while the droplets without cells flow into the negative channel. Scale bar: $100 \mu \mathrm{m}$. e Comparison of the formed hydrogels without and with sorting after a Live/Dead assay. Scale bar: $100 \mu \mathrm{m}$. f A plot of the percentages of the droplets encapsulating zero, single and multiple cells without and with sorting

environment. We achieve this aim by incorporating alginate, a biocompatible polymer that can be physically crosslinked with divalent ions ${ }^{30}$, into the DEX-rich phase. Through collecting the double emulsions into a calcium chloride $\left(\mathrm{CaCl}_{2}\right)$ solution bath, the released core droplets can be rapidly crosslinked to form spherical and uniformsized hydrogels (Fig. 4a, b).

Moreover, the transient use of the oil phase enables inclusion of a droplet sorting step after double-emulsion generation and before extraction of the core droplets to produce single-cell-laden hydrogels, which is difficult to achieve in other ATPS droplet generation approaches ${ }^{31}$. We demonstrate this process in an integrated channel composed of both generation and sorting parts (Fig. 4c). Here, a spacing oil flow is introduced to further gap the continuously generated droplets for accurate sorting, and the two branch channels are designed with precise differences in hydraulic resistances. As a result, the droplets are guided into the lower channel when no electric field is applied and are driven into the upper channel when an electric field is applied (Fig. 4d). Through setting the flow rates of the core, shell, generation and spacing oil phases 
at $200,600,3000$ and $3000 \mu \mathrm{L} / \mathrm{h}$, respectively, highthroughput sorting can be achieved at frequencies above $300 \mathrm{~Hz}$. To demonstrate the sorting-based fabrication of single-cell-laden microgels, the average cell number in each droplet is tuned to 0.1 so that only approximately $10 \%$ of the generated droplets contain single cells following the Poisson distribution. With a sorting step to remove the negative droplets, the single-cell encapsulation ratio increases to over $90 \%$, thus allowing a highthroughput analysis of the encapsulated single cells (Fig. $4 \mathrm{e}, \mathrm{f})$. Moreover, to verify the biocompatibility of this technique, a live/dead assay is performed on the encapsulated cells and shows high viability, with over $95 \%$ of the cells remaining alive. This result suggests that the encapsulated cells can be further cultured for tissue development and engineering applications.

\section{Discussion}

The core concept of this technique is to first use oil as a continuous phase to rapidly generate W-W-O doubleemulsion drops and then remove the oil to release the inner cores for water-in-water droplet formation. With the use of oil as a continuous phase, sorting can be further incorporated after the formation of double-emulsion drops and before the extraction of core droplets to select the candidates of interest.

In double-emulsion generation, the low interfacial tension that is frequently encountered for water-in-water droplets can be overcome by introducing an oil phase, thus achieving high levels of throughput and monodispersity in both passive and active methods. Through adjusting the flow rates, the morphology of the doubleemulsion drops formed can be precisely controlled, and the generation frequency can be as high as $\sim 2.4 \mathrm{kHz}$, which is 20 times higher than that of existing methods. Moreover, the fabricated double-emulsion drops can be used to form ATPS microcapsules by adding hydrogel components into the shell phase, which may serve as biocompatible carriers in drug delivery and food processing assays ${ }^{32}$.

The approach of active double-emulsion formation through sequential droplet injection is efficient for performing controlled reactions within the inner droplets, which is not limited to the demonstrated sample mixing and can be extended to other functional operations. For instance, through the incorporation of different individual cells by sequential injections, cell-to-cell communications and interactions at the single-cell level can be studied within biocompatible compartments. Moreover, if we further increase the gap between each injector or perform sequential injections in separate devices, the core droplet can be stabilized before subsequent injection, thus forming double emulsions with multiple separate cores. This type of structure is potentially applied in fabricating cell-like biomimetic architectures, such as protocells, where the core droplets can function as independent artificial organelles.

To gently break the double emulsions without damaging the inner cores, an oil evaporation-based method is introduced, enabling fabrication of monodisperse ATPS droplets with spherical shapes. The obtained all-aqueous microspheres can serve as biocompatible templates to perform various assays, as demonstrated in the microgel particle fabrication and DNA partitioning studies. Moreover, the employed core-release method is useful for recovering single-emulsion drops, which can be functionalized to extract the encapsulated single cells from water-in-oil droplets ${ }^{4}$.

When combined with accurate droplet sorting, the demonstrated production of single-cell-laden hydrogels promises further high-throughput analysis of the encapsulated single cells, such as detecting their immune responses to study the distribution of antibiotic resistance and monitoring their differentiation to probe the heterogeneity among populations. Moreover, noninvasive operations guarantee high cell viability, enabling further culture to develop microgels into functional building blocks for bottom-up tissue engineering ${ }^{33}$.

Despite the high efficiency of this method in generating and sorting ATPS droplets, there is still room to further exploit its potential. For instance, the finally formed droplets are still in a single-emulsion format, which restricts their use in applications that require controlled sample encapsulation and release ${ }^{34}$. Thus, further development should focus on the fabrication of more functional aqueous multiple-emulsion drops, which may be achieved by the introduction of additional immiscible aqueous flows for compartmentation.

In summary, the introduction of a transient oil medium enables high-throughput generation and sorting of waterin-water droplets, which can be used as templates to encapsulate single cells in hydrogel microspheres. The simplicity, high throughput and capacity to be integrated into more sophisticated microfluidic processes inspire new ways to use these droplets in various material synthesis and biological analyses.

\section{Materials and methods \\ ATPS droplet generation}

To prepare ATPS solutions, PEG $\left(M_{w} 8000\right.$, SigmaAldrich, USA $)$ and DEX $\left(\mathrm{M}_{\mathrm{w}} 10,000\right.$, Sigma-Aldrich, USA) polymers were dissolved in deionized water at weight ratios from 10 to $20 \%$. After centrifugation at $8000 \mathrm{rpm}$ for $1 \mathrm{~h}$, the solutions were left standing overnight to achieve complete separation. Then, the equilibrated PEG-rich and DEX-rich solutions were collected separately, and the polyelectrolytes, PSS and PAH (SigmaAldrich, USA), were dissolved into the two phases at 
concentrations of $2 \mathrm{mg} / \mathrm{mL}$. For further fluorescent labeling in the sequential injection studies, fluorescein isothiocyanate-dextran $\left(M_{w} 10,000\right.$, Sigma-Aldrich, USA $)$ was dissolved into the DEX-rich phase at a weight ratio of $0.5 \%$. A fluorinated oil (HFE7500, 3M, USA) was used as the continuous phase for droplet generation, and the weight ratios of a fluorosurfactant (008, RAN Biotechnologies, USA) were varied from 0.005 to $1 \%$. To form alginate hydrogels, an alginic acid sodium salt from brown algae (low viscosity, Sigma-Aldrich, USA) was dissolved into the DEX-rich phase at a weight ratio of $1 \%$, and a $1 \%$ weight ratio of $\mathrm{CaCl}_{2}$ solution (Aladdin, $\mathrm{CN}$ ) was used to extract the core droplets.

\section{Device fabrication}

The microfluidic devices were fabricated using a typical soft lithography replica molding technique ${ }^{35}$. First, a channel mold was fabricated on a silicon wafer (N100, University, USA) with SU-8 photoresist (2025, MicroChem, USA) using maskless lithography (SF-100 Xcel, Intelligent Micro Patterning, LLC, USA). Then, the PDMS prepolymer base (Sylgard 184, Dow Corning, USA) was crosslinked with the curing agent at a weight ratio of 10:1. After sufficient mixing by a conditioning mixer (AR-100, THINKY, JP), the mixture was poured onto a channel mold and cured at $65^{\circ} \mathrm{C}$ for $4 \mathrm{~h}$. Subsequently, the PDMS channel was peeled off from the mold. Finally, after inlet and outlet punching, the PDMS channel was bonded to a glass substrate (ISOLAB, DE) through oxygen plasma treatment (PDC-002, Harrick, USA) and heated at $90^{\circ} \mathrm{C}$ for $2 \mathrm{~h}$. The height, width and length of the generation channel were designed to be 65,120 and $2000 \mu \mathrm{m}$, respectively, and the whole channel was treated with a hydrophobic agent (Aquapel, PPG, USA) to guarantee stable droplet generation. To fabricate the electrodes required in active generation and sorting, empty channels were fabricated in the desired shape of the electrodes. Then, a low-melting-point metal wire (52225, Indium Incorporation, USA) was inserted into the electrode channel from one end at $95^{\circ} \mathrm{C}$, and negative pressure was applied to the other end to fill the whole channel with liquid metal. After further cooling the device to solidify the metal, metallic electrodes could be formed according to the desired shape.

\section{Cell assay}

An esophageal cancer cell line (KYSE-150) was used as the target encapsulant to test the sorting efficiency. For fluorescence labeling, the cells were transduced with lentiviral particles encoding green fluorescent protein (GFP). The coding sequence was cloned into a pLVX-puro backbone (Clontech, USA) following an EF-2 alpha promoter ${ }^{36}$. The cells were maintained in Roswell Park Memorial Institute (RPMI) 1640 Medium (Life Technologies, USA), $100 \mathrm{U} / \mathrm{mL}$ penicillin and $100 \mu \mathrm{g} / \mathrm{mL}$ streptomycin (Gibco,
USA) in a humidified atmosphere at $37^{\circ} \mathrm{C}$ with $5 \% \mathrm{CO}_{2}$. After trypsin treatment, the cells were transferred into the prepared DEX-alginate solution at a concentration of $3.05 \times 10^{6} / \mathrm{mL}$. The cell viability after encapsulation and sorting was tested using a Live/Dead reagent (calcein AM/ ethidium homodimer-1, Sigma-Aldrich, USA).

\section{Droplet sorting}

A typical florescence-activated droplet sorting (FADS) system was used to sort the double-emulsion drops with single cells ${ }^{37,38}$. A diode-pumped solid-state (DPSS) laser source $(20 \mathrm{~mW}, \mathrm{CNI}, \mathrm{CN})$, with a wavelength of $488 \mathrm{~nm}$, was used to excite the fluorescence signal. Guided by a dichroic mirror (Semrock, USA), the laser beam was reflected into a plan fluorite objective (Olympus, JP) and focused into the microfluidic channel. Then, the excited fluorescence signal propagated backward and was separated into two beams through a beam splitter (Thorlabs, USA), with $90 \%$ of the light received by a photomultiplier tube (PMT, Hamamatsu, JP) for fluorescence detection and the remaining $10 \%$ used for high-speed imaging (Phantom V9.1, Vision Research, USA). Subsequently, the triggered signal was processed by a custom LabVIEW program (PCIe 7842R, National Instruments, USA), responding with output signals applied to the electrodes after amplification by a high-voltage amplifier (5/80, Trek, USA).

\section{Acknowledgements \\ This work was supported by the General Research Fund (Nos. 17307919, 17329516, 17304017, 17305518, and 17306315) and the Research Impact Fund (No. R7072-18) from the Research Grants Council of Hong Kong, Excellent Young Scientists Fund (Hong Kong and Macau) (21922816) from the National Natural Science Foundation of China (NSFC) as well as the Seed Fund for Basic Research (Nos. 201811149241, 201711159249, and 201611159205), the Seed Fund for Translational and Applied Research (No. 201711160016) and Platform Technology Funding from the University of Hong Kong. We thank Prof. Maria Li Lung from HKU's Department of Clinical Oncology for providing the KYSE- 150 cell samples, Ph.D. candidate Yage Zhang for the assistance in cell culture, and Dr. Youchuang Chao for the discussion of ATPS solution preparation.}

\section{Author contributions}

L.N. and H.C.S. conceived the project, and Y.C. prepared the ATPS and cell samples. S.Y. designed and fabricated the devices. L.N. performed the experiments, and Y.C. analyzed the results. L.N. and H.C.S. wrote the manuscript.

\section{Conflict of interest}

The authors declare that they have no conflict of interest.

Supplementary information accompanies this paper at https://doi.org/ 10.1038/s41378-020-0180-0.

Received: 25 January 2020 Revised: 17 April 2020 Accepted: 6 May 2020 Published online: 07 September 2020

\footnotetext{
References

1. Shang, L., Cheng, Y. \& Zhao, Y. Emerging droplet microfluidics. Chem. Rev. 117, 7964-8040 (2017).

2. $\mathrm{Yu}, \mathrm{Y}$. et al. Microfluidic lithography of bioinspired helical micromotors. Angewandte Chemie 129, 12295-12299 (2017).
} 
3. Yu, Y., Guo, J., Sun, L., Zhang, X. \& Zhao, Y. Microfluidic generation of microsprings with ionic liquid encapsulation for flexible electronics. Research 2019 6906275 (2019).

4. Nan, L., Lai, M. Y. A., Tang, M. Y. H., Chan, Y. K., Poon, L. L. M. \& Shum, H. C. On-demand droplet collection for capturing single cells. Small. 16, 1902889 (2020).

5. Fidalgo, L. M. et al. From microdroplets to microfluidics: selective emulsion separation in microfluidic devices. Angewandte Chemie 120, 2072-2075 (2008).

6. Song, Y. et al. Fabrication of fibrillosomes from droplets stabilized by protein nanofibrils at all-aqueous interfaces. Nat. Commun. 7, 12934 (2016).

7. Chan, Y. K., Yan, W. H., Hung, L. T., Chao, Y., Wu, J. \& Shum, H. C. All-Aqueous Thin-Film-Flow-Induced Cell-Based Monolayers. ACS Appl. Mat. Interfaces. 11 22869-22877 (2019).

8. Chao, Y. \& Shum, H. C. Emerging aqueous two-phase systems: from fundamentals of interfaces to biomedical applications. Chem. Soc. Rev. 49, 114-142 (2020).

9. Frampton, J. P., White, J. B., Abraham, A. T. \& Takayama, S. Cell co-culture patterning using aqueous two-phase systems. J. Visual. Exp. 73, e50304 (2013)

10. Song, Y. et al. Budding-like division of all-aqueous emulsion droplets modulated by networks of protein nanofibrils. Nat. Commun. 9, 2110 (2018).

11. Dewey, D. C., Strulson, C. A., Cacace, D. N., Bevilacqua, P. C. \& Keating, C. D. Bioreactor droplets from liposome-stabilized all-aqueous emulsions. Nat. Commun. 5, 4670 (2014)

12. Chao, Y., Mak, S. Y., Rahman, S., Zhu, S. \& Shum, H. C. Generation of high-order all-aqueous emulsion drops by osmosis-driven phase separation. Small $\mathbf{1 4}$ 1802107 (2018)

13. Moon, B.-U., Abbasi, N., Jones, S. G., Hwang, D. K. \& Tsai, S. S. Water-in-water droplets by passive microfluidic flow focusing. Anal. Chem. 88, 3982-3989 (2016).

14. Hardt, S. \& Hahn, T. Microfluidics with aqueous two-phase systems. Lab Chip 12, 434-442 (2012).

15. Ma, Q. et al. Partitioning-dependent conversion of polyelectrolyte assemblies in an aqueous two-phase system. Soft Matter 14, 1552-1558 (2018).

16. Mak, S. Y., Chao, Y., Rahman, S. \& Shum, H. C. Droplet formation by rupture of vibration-induced interfacial fingers. Langmuir 34, 926-932 (2017).

17. Zhou, C. et al. Microfluidic generation of aqueous two-phase-system (ATPS) droplets by oil-droplet choppers. Lab Chip 17, 3310-3317 (2017).

18. Caen, O. et al. High-throughput multiplexed fluorescence-activated droplet sorting. Microsyst. Nanoeng. 4, 33 (2018).

19. Yuan, $\mathrm{H}$. et al. Picoinjection-enabled multitarget loop-mediated isothermal amplification for detection of foodborne pathogens. Anal. Chem. 90 13173-13177 (2018)

20. Moon, B.-U., Jones, S. G., Hwang, D. K. \& Tsai, S. S. Microfluidic generation of aqueous two-phase system (ATPS) droplets by controlled pulsating inlet pressures. Lab Chip 15, 2437-2444 (2015).
21. Liu, H. T. et al. A microfluidic strategy for controllable generation of water-inwater droplets as biocompatible microcarriers. Small 14, 1801095 (2018).

22. Mazutis, L. et al. Single-cell analysis and sorting using droplet-based microfluidics. Nat. Protoc. 8, 870-891 (2013).

23. Wang, $\mathrm{H}$. et al. One-step generation of core-shell gelatin methacrylate (GelMA) microgels using a droplet microfluidic system. Adv. Mater. Technol. 4 1800632 (2019).

24. Abate, A. \& Weitz, D. High-order multiple emulsions formed in poly (dimethylsiloxane) microfluidics. Small 5, 2030-2032 (2009).

25. Abate, A. R., Hung, T., Mary, P., Agresti, J. J. \& Weitz, D. A. High-throughput injection with microfluidics using picoinjectors. Proc. Natl Acad. Sci. USA 107, 19163-19166 (2010)

26. Rotem, A. et al. Single-cell ChIP-seq reveals cell subpopulations defined by chromatin state. Nat. Biotechnol. 33, 1165 (2015).

27. Mao, A. S. et al. Deterministic encapsulation of single cells in thin tunable microgels for niche modelling and therapeutic delivery. Nat. Mater. 16, 236 (2017).

28. Nan, L., Yang, Z., Lyu, H., Lau, K. Y. Y. \& Shum, H. C. A microfluidic system for one-chip harvesting of single-cell-laden hydrogels in culture medium. Adv. Biosyst. 3, 1900076 (2019).

29. Martinez, C. J. et al. A microfluidic approach to encapsulate living cells in uniform alginate hydrogel microparticles. Macromol. Biosci. 12, 946-951 (2012).

30. Lee, K. Y. \& Mooney, D. J. Hydrogels for tissue engineering. Chem. Rev. 101 1869-1880 (2001)

31. Ziemecka, I. et al. Monodisperse hydrogel microspheres by forced droplet formation in aqueous two-phase systems. Lab Chip 11, 620-624 (2011).

32. Shum, H. C., Kim, J.-W. \& Weitz, D. A. Microfluidic fabrication of monodisperse biocompatible and biodegradable polymersomes with controlled permeability. J. Am. Chem. Soc. 130, 9543-9549 (2008).

33. Khademhosseini, A. \& Langer, R. A decade of progress in tissue engineering. Nat. Protoc. 11, 1775 (2016)

34. Datta, S. S. et al. 25th anniversary article: double emulsion templated solid microcapsules: mechanics and controlled release. Adv. Mater. 26, 2205-2218 (2014).

35. Qin, D., Xia, Y. \& Whitesides, G. M. Soft lithography for micro-and nanoscale patterning. Nat. Protoc. 5, 491 (2010).

36. $Y u, V$. Z. et al. Nuclear localization of DNAJB6 is associated with survival of patients with esophageal cancer and reduces AKT signaling and proliferation of cancer cells. Gastroenterology 149, 1825-1836.e1825 (2015).

37. Baret, J.-C. et al. Fluorescence-activated droplet sorting (FADS): efficient microfluidic cell sorting based on enzymatic activity. Lab Chip 9, 1850-1858 (2009).

38. Tang, M. Y. \& Shum, H. C. One-step immunoassay of C-reactive protein using droplet microfluidics. Lab Chip 16, 4359-4365 (2016). 